\title{
PRC1 is taking the lead in PcG repression
}

Wiam Merini and Myriam Calonje*

Institute of Plant Biochemistry and Photosynthesis (IBVF-CSIC-University of Seville) Avenida América Vespucio, 49, Isla de La Cartuja, 41092 Seville, Spain

*For correspondence:

Myriam Calonje

email: myriam.calonje@ibvf.csic.es; Tel: +34 954489591; Fax: +34 954460165

Running title: PRC1-mediated gene repression

Key works: Arabidopsis, Plant development, Polycomb Group, PRC1, gene repression, H2A monoubiquitination, chromatin compaction

The total word count: 9164 words

Summary: 110 words

Significance statement: 39 words

Introduction: 473 words

Sections of the review: 5109 words

Future directions: 273 words

Acknowledgements: 24 words

References: 2814 words

Figure Legends: 210 words 


\section{Summary}

Polycomb group (PcG) proteins constitute a major epigenetic mechanism for gene repression throughout the plant life. For a long time, the PcG mechanism has been proposed to follow a hierarchical recruitment of $\mathrm{PcG}$ repressive complexes (PRCs) to target genes in which the binding of PRC2 and the incorporation of H3 lysine 27 trimethyl marks led to recruitment of $\mathrm{PRC} 1$, which in turn mediated $\mathrm{H} 2 \mathrm{~A}$ monoubiquitination. However, recent studies have turned this model upside-down by showing that PRC1 activity can be required for PRC2 recruitment and H3K27me3 marking. Here, we review the current knowledge on plant PRC1 composition and mechanisms of repression, as well as its role during plant development.

\section{Significance statement}

Recent studies on PRC1 function challenge the classical hierarchical model for recruitment of PcG complexes to target genes to mediate gene repression in Arabidopsis by showing that the binding of PRC1 triggers the recruitment of PRC2 and H3K27 trimethylation.

\section{Introduction}

PcG machinery plays a crucial role in many biological processes of higher eukaryotes, including development, differentiation and proliferation. PcG proteins are chromatin factors that maintain the transcriptional repression of their target genes. These proteins associate in multimeric complexes with different histone modifying activities (reviewed in Schwartz and Pirrotta, 2013). The two best characterized complexes are the PRC1, which mediates histone $\mathrm{H} 2 \mathrm{~A}$ lysine 119 monoubiquitination (H2Aub) of its target genes (Wang et al., 2004; de Napoles et al., 2004; Lagarou et al., 2008; Gutiérrez et al., 2012; 
Bratzel et al., 2010) and PRC2, which has histone $\mathrm{H} 3$ lysine 27 (H3K27) trimethyltransferase activity (Cao et al., 2002; Czermin et al., 2002; Müller et al., 2002; Chanvivattana et al., 2004; Schubert et al., 2006). Numerous studies have demonstrated a tight link between PRC1 and PRC2; accordingly, the combined activity of the two complexes is required for stable repression of the target genes, but, how this is exactly accomplished is far from clear.

PRC1 core components were initially identified in flies. Drosophila melanogaster (from now on Drosophila) PRC1 includes Polycomb (Pc), which can bind H3K27me3 marks through its chromodomain; Polyhomeotic $(\mathrm{Ph})$, which function in the complex is well known; Posterior sex comb (Psc) or its paralog $\mathrm{Su}(\mathrm{z}) 2$ that can inhibit transcription and mediate chromatin compaction in addition to forming an E3 monoubiquitin ligase module with the forth core component, dRing1 (also known as Sex combs extra (Sce)), to mediate H2A monoubiquitination (Shao et al., 1999; Saurin et al., 2001; Francis et al., 2001; Peterson et al., 2004) (Figure 1). These proteins have several homologs in vertebrates (Levine et al., 2002). The Chromodomain protein family (CBX2, CBX4, CBX6, CBX7 and CBX8) carries out Pc function in mammals (Morey et al., 2012). Mammalian homologs of $\mathrm{Ph}$ are $\mathrm{PH} 1, \mathrm{PH} 2$, and PH3 (Tonkin et al., 2002); homologs of dRing are named RING1A and RING1B (Schoorlemmer et al., 1997); and finally, BMI1, NSPC1, MEL18, PCGF3, PCGF5 and MBLR, which collectively are named the PcG RING fingers (PCGFs), are the homologs of Psc (Gao et al., 2012; Calonje, 2014) (Figure 1).

About a decade ago and according to several functional and molecular evidences, PcGmediated repression in animals was proposed to follow a hierarchical recruitment of PcG complexes to target genes: first, PRC2 incorporates $\mathrm{H} 3 \mathrm{~K} 27 \mathrm{me} 3$ at a specific gene, and then PRC1 is recruited by the ability of Pc to bind to H3K27me3. Once recruited, 
PRC1 mediates histone $\mathrm{H} 2 \mathrm{~A}$ monoubiquitination to maintain stable repression (Wang et al., 2004). The hierarchical model for PcG recruitment was extended to plants, even though at that time there were many gaps to understand the $\mathrm{PcG}$ repression mechanism in these organisms. However, recent data on PRC1 function in Arabidopsis thaliana (from now on Arabidopsis) have shed some light on the mechanism, revealing unexpected twists. Here, we review the current knowledge on plant PRC1 composition and mechanism of repression, as well as its role during plant development.

\section{PRC1 components and their loss-of-function mutants: a historic perspective}

The implication of the well-conserved plant PRC2 components in important developmental processes, such as the repression of flowering during vegetative development, the suppression of endosperm development in absence of fertilization, and the vernalization response, has for long been known (reviewed in Hennig and Derkacheva, 2009). In addition, clear evidences emerged soon indicating that PRC2mediated H3K27me3 was necessary for repression of key developmental genes (Chanvivattana et al., 2004; Schubert et al., 2006; Köhler, Hennig, Bouveret, et al., 2003; Köhler, Hennig, Spillane, et al., 2003); however, the participation of a plant PRC1 in the repression was questioned, since the identification of plant PRC1 components remained elusive for a long time (Figure 1).

The first proposed PRC1 component in plants was LHP1, also known as TERMINAL FLOWER 2 (TFL2). LHP1 was identified as a homolog of animal HETEROCHROMATIN PROTEIN 1 (HP1), which is required for heterochromatin maintenance (Gaudin et al., 2001). LHP1 contains a chromodomain and a chromo shadow domain like HP1 (Gaudin et al., 2001; Kotake et al., 2003) (Figure 1); however, LHP1 is usually localized in euchromatin and is needed for maintenance of gene 
repression in euchromatin (Libault et al., 2005; Nakahigashi et al., 2005); accordingly, LHP1 co-localizes with H3K27me3 in vivo (Turck et al., 2007; X., Zhang et al., 2007; Exner et al., 2009). Furthermore, lhpl mutants showed misexpression of some PRC2 target genes, most of which are involved in flowering pathways (Kotake et al., 2003; Libault et al., 2005; Nakahigashi et al., 2005), and an early flowering phenotype as mutant plants in PRC2 components (Kotake et al., 2003; Goodrich et al., 1997; LopezVernaza et al., 2012). Therefore, LHP1 seemed to play a similar role to Drosophila Pc, which led to propose a model in which LHP1 interacts with H3K27me3 marks and recruits a plant PRC1 for stable repression of PcG targets (Turck et al., 2008; X., Zhang et al., 2007). However, it has been shown recently that LHP1 co-purifies with PRC2 and that LHP1 assists the recruitment of PRC2 to target sites for re-establishing the levels of H3K27me3 after replication (Derkacheva et al., 2013), changing the view of LHP1 as a PRC1 component (reviewed in this issue by Lars Hennig and Claudia Kohler).

The second proposed PRC1 component was EMBRYONIC FLOWER 1 (EMF1) (Calonje et al., 2008). emfl mutants were described around twenty years ago as mutant plants that skip vegetative development and flower right after germination. The weak emf1-1 mutant produced a small inflorescence upon germination and a few flowers that were sterile. All leaves were small and petiole-less, thought to be cauline leaves. The strong emf1-2 mutant could not even produce inflorescences and flowers; all lateral organs eventually developed into carpels (Sung et al., 1992; Yang et al., 1995). Cloning of the EMF1 gene revealed that it encodes a novel protein with little homology to any gene of known function (Aubert et al., 2001). The identification of several motifs in EMF1 protein sequence suggested that it could function as a transcriptional regulator during shoot development (Moon et al., 2003; Sung et al., 2003). Accordingly, emf1 
mutants displayed ectopic expression of many different genes; among them were flower homeotic genes such as APETALA 1 (AP1), AP3, PISTILLATA (PI) and AGAMOUS (AG) (Moon et al., 2003). Interestingly, emfl-1 mutants showed exactly the same phenotype as mutant plants in the PRC2 component EMF2 and a high degree of overlap in gene misregulation (Moon et al., 2003), suggesting a cooperation of EMF1 and EMF2 in the repression of the flower homeotic genes during vegetative development (Chen et al., 1997; Moon et al., 2003), although the role of EMF1 in the process was not understood.

It was some years later when EMF1 was proposed as a plant specific PRC1 component (Calonje et al., 2008). It was found that EMF1 acted in concert with PRC2 in the repression of the flower homeotic gene $A G$ during vegetative development, displaying functional similarities to Drosophila Psc (Calonje et al., 2008). Drosophila Psc has a long intrinsically disordered C-terminal region (CTR) with patches of positive charged interspersed with local negatively charged regions. Psc-CTR has the ability to inhibit nucleosome remodeling, repress transcription and compact chromatin (Francis et al., 2001; Francis et al., 2004; Lo and Francis, 2010). Likewise, EMF1 has a long intrinsically disordered region with the same abilities (Calonje et al., 2008; Beh et al., 2012; Calonje, 2014) (Figure 1). A different PRC1 subunit in mammals, CBX2/M33, has also a charged region and possesses analogous biochemical properties to Psc-CTR, thus suggesting that CBX2/M33 may functionally replace the Psc-CTR function in mammalian PRC1 (Grau et al., 2011) (Figure 1). According to this, Psc-CTR activity seems to be conserved across eukaryotes, while the identity of the functional subunit may vary between taxa (Beh et al., 2012).

Interestingly, the levels of $\mathrm{H} 3 \mathrm{~K} 27 \mathrm{me} 3$ at $A G$ were reduced in emf1-2 mutants. However, at this time the hierarchical model for recruitment of PcG complexes in which PRC1 
acts downstream PRC2 was widely accepted, therefore, this finding was interpreted as a requirement of EMF1 for maintaining a stable histone methylation pattern of the target gene, thereby placing EMF1 activity downstream of EMF2 (Calonje et al., 2008).

Full-genome expression pattern analysis of emfl and emf2 mutants showed that both proteins regulate additional gene programs, including photosynthesis, seed development, hormone, stress, and cold signaling (Kim et al., 2010). Later on, genomewide localization of EMF1 binding and $\mathrm{H} 3 \mathrm{~K} 27$ me 3 modification in wild-type and emf12 plants showed a substantial number of genes marked with H3K27me3 and occupied by EMF1. Interestingly, around $60 \%$ of these genes showed decreased levels of H3K27me3 in emf1-2, indicating that there are genes that requires EMF1 for their H3K27me3 marking; however, there was a subset of genes that remained H3K27 trimethylated in absence of EMF1 (Kim et al., 2012). Beside these genes, EMF1 was bound also to a percentage of unmethylated genes. Among them, genes encoding proteins located in cytosol and chloroplast were over-represented. These genes tend to be actively transcribed genes with high RNA levels (Kim et al., 2012). Strikingly, it has been recently shown that animal PRC1 can participate in gene activation by orchestrating the 3D chromatin structure changes involved in transcriptional activation of their target genes, although the detailed molecular mechanisms at the basis of this phenomenon remain to be addressed (Kondo et al., 2014).

On the other hand, EMF1 has been shown to interact with different proteins in a yeast two-hybrid screening. These proteins were named EMF1 INTERACTING PROTEINS (EIPs) (Park et al., 2011). Among them, EIP1, EIP6 and EIP9 are proposed to interact independently with EMF1 during vegetative development to regulate flowering time in Arabidopsis (Park et al., 2011). EIP1, EIP6 and EIP9 are predicted to encode a WNK (with-no-lysine) kinase (EIP1/WNK8), a B-box zinc-finger protein and a DnaJ-domain 
protein. EIP1 was proposed to regulate EMF1 activity by phosphorylating some of the potential phosphorylation sites in EMF1; EIP6 contains a B-box domain at the Nterminus, which is also found in the floral inducer CONSTANS (CO) and seems to play a role in protein interaction (Torok and Etkin, 2001); and EIP9 was proposed to act together with EMF1 in mediating epigenetic gene regulation, since several DnaJ proteins have been reported to play roles in gene repression via chromatin modification (Park et al., 2011). Whether the interaction of these proteins with EMF1 is stable or transient it is not known.

Finally, the most recently identified PRC1 components in plants were the PRC1 RING finger proteins. Analyzing the domain structure of animal PRC1 RING finger protein family, which comprises both BMI1- and RING1-like proteins, it was found that this family of proteins has a conserved RING finger domain at the $\mathrm{N}$-terminus and a new Ubiquitin-like domain named RAWUL (Ring-finger And WD40 associated UbiquitinLike) at the C-terminus (Sanchez-Pulido et al., 2008; Bezsonova et al., 2009) (Figure 1). In Arabidopsis, there are five PRC1 RING finger homologs; two RING1-like (AtRING1A and AtRING1B) and three BMI1-like (AtBMI1A, AtBMI1B, and AtBMI1C) (Sanchez-Pulido et al., 2008) (Figure 1), but the functional conservation of these proteins to their animal counterparts was unclear at this time, as H2Aub chromatin marks had not been detected in plants (K., Zhang et al., 2007).

The analysis of atringla/b loss-of-function mutants indicated that these genes were involved in the repression of Class I KNOTTED-like homeobox (KNOX) transcription factors to suppress ectopic-meristem formation. In addition, atringla/b mutant plants showed an enlarged shoot apical meristem (SAM), resulting in enlarged stems and fasciated inflorescence meristems that produced heavily fused sterile flowers; therefore, loss-of-function of both AtRING1A and AtRING1B were proposed to affect initiation, 
maintenance, and differentiation of inflorescence and floral organs ( $\mathrm{Xu}$ and Shen, 2008). AtRING1 proteins were shown to interact with CURLY LEAF (CLF), a PRC2 core component catalyzing H3K27-methylation, and with LHP1, suggesting a model in which AtRING1A, AtRING1B, and LHP1 form a PRC1-like complex for transcriptional suppression of KNOX genes (Xu and Shen, 2008).

On the other hand, loss-of-function in AtBMI1A and B led to derepression of embryonic traits in vegetative tissues as a consequence of a strong misexpression of seed maturation genes (e.g. FUSCA 3 (FUS3), LEAFY COTYLEDON 1 (LEC1), ABSCISIC ACID INSENSITIVE 3 (ABI3)) and stem cell regulators (e.g. WUSCHEL (WUS), WUSCHEL-related homeobox 5 (WOX5)) (Bratzel et al., 2010). Interestingly, these mutants showed a very similar phenotype to the one displayed by mutant plants impaired in PRC2 function (Chanvivattana et al., 2004; Aichinger et al., 2009). Later on, deregulation of the embryonic program was also detected in atringla/b mutants (Chen et al., 2010), indicating that they regulate a common subset of targets.

In addition, AtBMI1A and AtBMI1B were shown to interact in vitro with LHP1 and EMF1 (Bratzel et al., 2010), and with the AtRING1s (Chen et al., 2010), suggesting their participation in a PRC1-like complex. More important, AtBMI1A and AtBMI1B were shown to mediate in vitro and in vivo $\mathrm{H} 2 \mathrm{~A}$ monoubiquitination. The in vitro activity was also demonstrated for AtRING1A and AtRING1B (Bratzel et al., 2010), revealing that $\mathrm{H} 2 \mathrm{~A}$ monoubiquitination takes place in plants and indicating that all Arabidopsis PRC1 RING finger homologs can act as E3 ubiquitin ligases (Bratzel et al., 2010).

Characterization of the third AtBMII gene, AtBMIIC, showed that, in contrast to AtBMI1A and B that are ubiquitously expressed, it was expressed during endosperm, stamen and root development (Bratzel et al., 2012; Li et al., 2011). Surprisingly, 
AtBMI1C was found to be an imprinted gene maternally expressed in the endosperm but biallelically expressed in stamen and roots (Bratzel et al., 2012). Nevertheless, AtBMIC displayed in vitro $\mathrm{H} 2 \mathrm{~A}$ monoubiquitin ligase activity and interacted with other PRC1 components as the other AtBMI1s (Bratzel et al., 2012). Furthermore, the progeny of atbmila $\mathrm{I}^{-} \mathrm{b}^{-/-} \mathrm{c}^{+/-}$plants showed aborted seeds and seedlings that displayed a phenotype similar to $a t b m i l a / b$ in the aerial part but with stronger defects in the root, indicating that AtBMI1 proteins are required during seed and vegetative development, acting at least partially redundantly in the tissue where they are co-expressed (Yang et al., 2013).

In addition, it has been found that $A t B M I I C$ overexpression driven by the $35 S$ promoter caused increased levels of $\mathrm{H} 2 \mathrm{~A}$ monoubiquitination that resulted in an early flowering phenotype. Accordingly, suppression of the flowering repressor FLOWERING LOCUS $C(F L C)$ and activation of the flowering promoter gene FLOWERING LOCUS T (FT) were observed in overexpressing lines, which suggest the participation of AtBMI1 proteins in flowering time control by regulating the expression of FLC (Li et al., 2011). Consistent with a possible role of the PRC1 RING finger proteins in regulating flowering time, it has been recently shown that loss-of-function of AtRING1A causes late-flowering phenotype due to misexpression of two floral repressors, MADS AFFECTING FLOWERING 4/5 (MAF4/5), which in turn repress two floral pathway integrators, FT and SUPPRESSOR OF OVEREXPRESSION OF CONSTANS 1 (SOC1) (Shen et al., 2014).

Plant homologs to animal PRC1 core component $\mathrm{Ph}$ have not been identified. It might be possible that a yet unknown plant-specific protein under takes Ph role, like EMF1 has assumed some of Psc functions. This, together with the fact that LHP1 displays a non-conserved role in plants participating in PcG repression (Derkacheva et al., 2013), 
indicates important differences between animal and plant PcG mechanism. Nevertheless, it is now clear that PRC1 function plays a crucial role in important developmental processes in plants. How the different components may associate with each other to display PRC1 function in these organisms will be discussed in the following section.

\section{PRC1 in plants: one or more than one complex?}

atringl or atbmil single mutants display a wild type-like phenotype and only combination of atringl $a$ and $b$ or atbmil $a$ and $b$ mutants shows a phenotype, indicating that AtRING1A/B and AtBMI1A/B can act redundantly in the regulation of gene expression (Bratzel et al., 2010; Chen et al., 2010); however, whether each AtBMI1 or AtRING1 has additionally specific target loci or acts in a tissue specific manner is still unknown. The fact that AtBMIC displays lower sequence similarity to AtBMI1A or AtBMI1B than the one displayed by AtBMI1A and AtBMI1B (Bratzel et al., 2010; Chen et al., 2010), suggests functional difference between AtBMIC and the other two AtBMI1s. Indeed, while AtBMI1A and B are able to carry out AtBMI1C function in the root, AtBMIC is only able to partially suppress the root defects in atbmila/b mutants (Bratzel et al., 2010; Yang et al., 2013), supporting a partial overlapping function.

In mammals, six forms of PRC1 have been identified. All contain one RING1 protein, either RING1B or the closely related protein RING1A, and one of the six PCGFs (BMI1, NSPC1, MEL18, PCGF3, PCGF5, MBLR) (Gao et al., 2012). In vitro studies have established that RING1B alone shows poor E3 activity on nucleosomes and that BMI1 has no in vitro activity, but that the RING1B-BMI1 heterodimer displays increased E3 ligase activity (Buchwald et al., 2006; Li et al., 2006; Bentley et al., 2011). Similarly, Drosophila dRing-Psc module has been shown to display in vitro H2A 
E3 ligase activity (Lagarou et al., 2008). Accordingly, the RING1-PCGF module has been considered the framework on which the different PRC1s are assembled (reviewed in Gil and O'Loghlen, 2014). However, it is not clear whether the same module is formed in Arabidopsis, since the two AtRING1 and the three AtBMI1 proteins display in vitro activity on H2A.1 (Bratzel et al., 2010; Bratzel et al., 2012). In addition, plants mutant for $A t B M I I A / B$ showed a significant global decrease of $\mathrm{H} 2 \mathrm{Aub}$, which is reflected in a decrease of this mark at the transcriptional start site region of specific targets, furthermore, this modification was undetectable in atbmila/b/c mutants (Yang et al., 2013), indicating that the AtBMI1s are crucial for the in vivo activity.

The in vivo $\mathrm{H} 2 \mathrm{~A}$ monoubiquitination activity of AtRING1A/B has not been tested yet, thus it is not known how these proteins affect $\mathrm{H} 2 \mathrm{Aub}$ levels. It might be possible that the AtBMI1 proteins confer activity to the complex and AtRING1 modulate the activity, alternatively, that both AtBMI1 and AtRING1 proteins are in vivo active H2A E3 ubiquitin ligases. Interestingly, loss-of function in AtRING1 leads to a weaker derepression of embryonic traits during seedling development than loss-of-function in AtBMI1, and atbmila/b mutants do not display the highly fasciated stem and the heavily fused flowers phenotype observed in atringla/b (Xu and Shen, 2008; Bratzel et al., 2010), raising the possibility of functional complexes containing AtBMI1 or AtRING1 homodimer.

Recently, the crystal structure of the human RING1B-BMI1 together with the E2 enzyme UbcH5c bound to a nucleosome has been resolved (McGinty et al., 2014). The authors found that the arginine in position 98 of RING1B is primarily responsible for binding to the nucleosome. Mutating this residue resulted in a 50-fold decrease in binding affinity and H2A monoubiquitination. The BMI1 subunit seems to make a lesser contribution to nucleosome binding affinity, although contact with the amino- 
terminal helix of $\mathrm{H} 3$ is crucial for binding, and presumably also for fixing the orientation of the complex on the nucleosome surface (McGinty et al., 2014; Müller and Müller, 2014). Conservation of these key residues in the Arabidopsis PRC1 RING finger proteins could provide some clues to determine if homo and hetero dimers can be formed in plants. This possibility would increase the number of PRC1 variants in Arabidopsis. In any case, interactions among different AtBMI1s and AtRING1s have been reported (Bratzel et al., 2010; Chen et al., 2010), which support several possible combinations.

The different PRC1 RING fingers might show preference for distinct $\mathrm{H} 2 \mathrm{~A}$ variants. Arabidopsis contains 13 genes (HTAs) that encode different H2A variants. The canonical H2A variants are HTA1, HTA2, HTA10, and HTA13 (H2A.6, H2A.3, H2A.1 and H2A.2, respectively); HTA3 and HTA5 (H2AXB and H2AXA) have the conserved C-terminal sequence characteristic of H2A.X variants; HTA6, HTA7, and HTA12 (H2A.7, H2A.5 and H2A.4) display a C-terminal region distinctive of these group of plant histones; and HTA8, HTA9, and HTA11 (H2AV2, H2AV3 and H2AV1) share a high homology with H2A.Z variants from other organisms (March-Díaz and Reyes, 2009). It has been proposed that the structural differences confer to $\mathrm{H} 2 \mathrm{~A}$ variants distinctive features for specific functions within chromatin. Whether these functions are regulated by posttranslational modifications remains to be investigated. So far, only canonical H2A variants have been shown to be in vivo target of AtBMI1 (Bratzel et al., 2010; Yang et al., 2013), however, according to the current picture in animals (Sevilla and Binda, 2014), the function of some $\mathrm{H} 2 \mathrm{~A}$ variants may be regulated by modification in plants.

On the other hand, when comparing genes that are mis-expressed in the different PRC1 mutants, it is surprising to find that there are genes misregulated in emfl but not in 
atbmil or atringl and vice versa, which raises questions like how many functional PRC1s exist in Arabidopsis and what is the biological relevance for such diversification.

As we mentioned before, Drosophila Psc-CTR has the ability to inhibit nucleosome remodeling, repress transcription and compact chromatin (Francis et al., 2001; Francis et al., 2004; Lo and Francis, 2010); abilities that seem independent of Psc implication in H2Aub monoubiquitination (Lagarou et al., 2008) Analysis of Drosophila mutants lacking individual PRC1 subunits revealed the existence of a subset of PcG targets that are deregulated in absence of Psc but remains repressed in the absence of the dRing, while other target genes require all four PRC1 subunits for repression; therefore, the repression of the first type of targets may rely on Psc-CTR, and the repression of the second type of targets on H2A monoubiquitination (Gutiérrez et al., 2012). In Arabidopsis, Psc activities are carried out by different proteins: the AtBMI1s that are involved in H2A monoubiquitination (Sanchez-Pulido et al., 2008; Bratzel et al., 2010), and EMF1 that is the functional equivalent to Psc-CTR (Calonje et al., 2008; Beh et al., 2012) (Figure 1). A recent report shows the localization of H2Aub marks in a subset of PcG targets and the effect of different PcG mutations on H2Aub deposition. According to the role of AtBMI1 proteins, atbmil mutants displayed decreased levels of H2Aub marks at seed maturation genes. Conversely, loss of function of EMF1 did not alter significantly the levels of H2Aub at these genes (Yang et al., 2013). On the other hand, there were other EMF1 targets, such as flower homeotic gene $A G$, in which the H2Aub marks were not detected (Yang et al., 2013). Consistent with these results, emfl-2 mutants did not display a significant derepression of the seed maturation genes (Yang et al., 2013), and atbmil or atringl mutants did not misexpress flower homeotic genes (Xu and Shen, 2008; Bratzel et al., 2010). Therefore, it seems that in Arabidopsis there are also two types of PRC1-mediated repression, the H2Aub-dependent and the H2Aub- 
independent repression, which requires EMF1. Since in Arabidopsis AtBMI1 and Psc activities are not associated in the same protein, it might be possible that different PRC1s carry out the different types of repression. However, there are several lines of evidence suggesting that this may not be the case. First, there are target genes that require both the PRC1 RING fingers and EMF1 for repression, as it is the case of FLC and MAF4/5 (Kim et al., 2010; Li et al., 2011; Molitor et al., 2014); second, EMF1 in vitro interacts with the PRC1 RING finger proteins (Bratzel et al., 2010; Bratzel et al., 2012); and third, EMF1 in vivo is bound to genes regulated by the PRC1 RING fingers (Kim et al., 2012). Therefore, EMF1 and the PRC1 RING finger may coexist in the same complex despite the observation that PRC1-mediated repression varies depending on the target gene. Presumably, association of different subunits determines the specificity and type of repression. The in vivo purification of PRC1 will be required to clarify this point.

\section{PRC1 comes first in PcG repression}

PcG repression to specific targets has been widely thought to require first the incorporation of $\mathrm{H} 3 \mathrm{~K} 27 \mathrm{me} 3$ marks by PRC2 and then the recruitment of PRC1 through the binding of Pc to H3K27me3 marks (Wang et al., 2004); according to this, PRC1 activity was proposed to stabilize the repression of H3K27me3 marked loci (Levine et al., 2004; Schuettengruber and Cavalli, 2009; Margueron and Reinberg, 2011).

However, studying the role of AtBMI1 proteins in PcG mediated repression in Arabidopsis, it was found that the lack of AtBMI1 activity caused a significant decrease in the levels of $\mathrm{H} 3 \mathrm{~K} 27 \mathrm{me} 3$ at seed maturation genes and that AtBMI1 activity was required for the initial repression of the genes (Yang et al., 2013). Furthermore, several evidences indicated EMF1 activity is required for $\mathrm{H} 3 \mathrm{~K} 27 \mathrm{me} 3$ marking at some target 
genes (Kim et al., 2012; Yang et al., 2013). In addition, the fact that EMF1 binding profile has the strongest signal around the transcriptional start site and then gradually declines towards the $3^{\prime}$ end, whereas $\mathrm{H} 3 \mathrm{~K} 27$ me3 produced by PRC2 forms a broader domain (Kim et al., 2012), did not fit with the idea of PRC1 coinciding with H3K27me3 marks. According to the new results, the binding and/or activity of PRC1, either H2Aub-dependent or -independent, may act as a docking point for PRC2 and subsequent spreading of $\mathrm{H} 3 \mathrm{~K} 27 \mathrm{me} 3$ marks at some target genes, which is turning the classic hierarchical model upside-down (Yang et al., 2013; Calonje, 2014) (Figure 2).

The requirement of PRC1 activity for $\mathrm{H} 3 \mathrm{~K} 27$ me3 marking was then confirmed in the repression of other genes, such as several seed developmental genes (e.g. DELAY OF GERMINATION 1 (DOG1)) during germination (Molitor et al., 2014), and MAF4 and MAF5 during the floral transition (Shen et al., 2014). Therefore, it seems that the PRC1dependent recruitment of $\mathrm{PRC} 2$ takes place at a considerable number of targets in Arabidopsis. Localization of $\mathrm{H} 3 \mathrm{~K} 27 \mathrm{me} 3$ marks in atbmil or atringl mutants will determine whether these genes represent the majority. However, there are also examples in which PRC1 and PRC2 activities are apparently recruited independently to the target genes, as is the case of the stem cell regulator WUS (Yang et al., 2013). In addition, it has been shown that EMF1 binds genes that lack H3K27me3 marks (Kim et al., 2012), suggesting a PRC2 independent role of EMF1. In any case, contrary to the previous idea, the binding of PRC1 can be independent on H3K27 methylation in Arabidopsis.

Surprisingly, three recent articles reached the same conclusion in vertebrates by showing that the binding of a variant PRC1 and subsequent $\mathrm{H} 2 \mathrm{~A}$ ubiquitination of surrounding chromatin is sufficient to trigger the recruitment of PRC2 and H3K27 trimethylation (Blackledge et al., 2014; Cooper et al., 2014; Kalb et al., 2014), 
indicating that the sequence of events in PcG repression is conserved in the two kingdoms, although in the reversed order than previously thought.

But, how can the PRC1-dependent PRC2 recruitment take place? PRC2 might be recruited to the target genes via interaction with PRC1 components or/and H2Aub. In mammals, it has been shown recently that nucleosomes containing H2Aub bind PRC2 components (Kalb et al., 2014). It is not known whether any of the PRC2 components can bind H2Aub marks in Arabidopsis but the PRC2 component CLF has been shown to physically interact with AtRING1A in yeast two-hybrid assays (Xu and Shen, 2008). In addition, the fact that LHP1 co-purifies with PRC2 and also interacts with several PRC1 components (Derkacheva et al., 2013; Xu and Shen, 2008; Wang et al., 2014), suggest that protein-protein interactions may be involved in the recruitment of PRC2.

It has been proposed that a positive feedback loop might occur at PcG target genes in which $\mathrm{H} 2 \mathrm{Aub}$ stimulates $\mathrm{H} 3 \mathrm{~K} 27 \mathrm{me} 3$, which at the same time facilitate the binding of PRC1 (Comet and Helin, 2014). In such mechanism, the ability of LHP1 to interact with PRC1, PRC2 and H3K27me3 marks may be crucial for extending the marks far from the recruitment site.

\section{Factors associated with PRC1 function in Arabidopsis}

PcG components are ubiquitously expressed; therefore, whether or not they are targeted to a given locus in a specific cell type or developmental stage has been a major research problem, even more considering that none of the PcG subunits can bind DNA in a sequence-specific fashion. In the classic hierarchical model, the recruitment of PRC1 was solved by the binding of Pc homologs (or the functional equivalent LHP1 in the case of Arabidopsis) to $\mathrm{H} 3 \mathrm{~K} 27 \mathrm{me} 3$ marks; however, according to recent data, the 
recruitment of PRC1 may take place independently of PRC2. Therefore, there may be associated factors involved in the specific targeting of PRC1.

Animal canonical PRC1s have a core of four subunits that have different paralogs, accordingly, different complexes have been described (reviewed in Schwartz and Pirrotta, 2013); however, a spate of recent publications has greatly expanded the range of PRC1 variants in which some canonical subunits have been replaced by other proteins, suggesting different biochemical properties of the variant complexes that may be involved in the specific recruitment (reviewed in Schwartz and Pirrotta, 2013). Consistent with this, the new PRC1 component RING1/YY1-binding protein (RYBP) has been proposed to bridge specific PRC1 variants to YY1(Tavares et al., 2012), the mammalian homolog of the Drosophila PRC1/2 recruiting factor Pleiohomeotic (Pho) (Klymenko et al., 2006; Simon and Kingston, 2009). In addition, the promoters of most PcG-regulated genes in mammals contain clusters of $\mathrm{CpG}$ dinucleotides, the $\mathrm{CpG}$ islands. The mammalian histone H3K36 demethylase KDM2B forms a complex with RING2 and PCGF1. KDM2B removes the dimethylation or trimethylation mark of $\mathrm{H} 3 \mathrm{~K} 36$ and binds to $\mathrm{CpG}$ islands through its $\mathrm{CXXC}$ domain, recruiting this PRC1 variant to DNA (Lagarou et al., 2008; Farcas et al., 2012; He et al., 2013; Wu et al., 2013). Therefore, the incorporation of factors that provide different biochemical properties to the PRC1 variants may be indeed important for targeting different subsets of genes and/or for context-dependent repression.

In Arabidopsis, all core subunits of animal PRC1 have not been identified yet; however, according to the current data, it seems that plants lack Ph and Pc sequence homologs; therefore, it might be possible that in plants only exist non-canonical PRC1s that associate with different proteins for recruitment (Figure 3). In fact, specific targeting of PRC1 has been reported for the seed maturation genes (Yang et al., 2013). According to 
this report, PRC1 is recruited to seed maturation genes by the VIVIPAROUS 1 (VP1)/ ABCISIC ACID INSENSTIVE 3 (ABI3)-Like (VAL1/2/3) transcription factors (Yang et al., 2013). Both VAL and AtBMI1 proteins suppress the seed maturation program after germination (Suzuki and McCarty, 2008; Guerriero et al., 2009; Bratzel et al., 2010); consequently, atbmila/b/c and vall/2 mutants display the same phenotype (Yang et al., 2013). The VAL proteins interact with AtBMI1A/B in vitro and are required for H2Aub marking of the seed maturation genes, placing VAL upstream of AtBMI1 activity in the repression. Interestingly, the VAL proteins, contain a B3 DNA binding domain that recognizes RY/Sph DNA elements (Suzuki et al., 2007; Suzuki and McCarty, 2008). Since the promoters of the seed maturation genes contain RY/Sph DNA elements, it has been proposed that the VAL proteins are involved in the specific recruitment of PRC1 to these genes (Yang et al., 2013). On the other hand, EMF1 directly interacts with the MADS-box domain transcription factor FLC and this association is required for FT repression, suggesting that FLC may recruit PRC1 to specific targets for repression (Wang et al., 2014). So far, no other DNA binding protein has been identified in Arabidopsis as PRC1 recruiter (Figure 3); however, it would not be surprising to find many other DNA binding factors, thus providing specificity to the complex.

Factors that interact with previously established histone modifications are proposed to play an important role in PcG recruitment (Figure 3). In fact, the VAL proteins, in addition to the $\mathrm{B} 3$ domain, have a plant homeodomain (PHD) and a $\mathrm{CW}$ domain that recognize H3K4me3 marks (Sanchez and Zhou, 2011; Hoppmann et al., 2011). The binding of VAL to $\mathrm{H} 3 \mathrm{~K} 4 \mathrm{me} 3$ marks through these domains been proposed to contribute in PRC1 targeting (Calonje, 2014). Furthermore, a recent report has proposed that the ALFIN1-like (ALs) proteins are involved in the recruitment of PRC1 (Molitor et al., 
2014). ALs are PHD finger proteins that bind H3K4me2/3 (Lee et al., 2009). This report shows that the PRC1 components AtBMI1 and AtRING1 physically interact with ALs and that the loss of AL6 and AL7 partly mimics the delay in germination phenotype of atbmila/b mutant, suggesting a model in which the AL-PRC1 complexes built around $\mathrm{H} 3 \mathrm{~K} 4 \mathrm{me} 3$ lead to a switch from the $\mathrm{H} 3 \mathrm{~K} 4 \mathrm{me} 3$-associated active to the H3K27me3-associated repressive transcription state of seed developmental genes (Molitor et al., 2014).

On the other hand, reprogramming of gene activity involves the removal of pre-existing chromatin marks and the incorporation of new modifications, which reflects a new transcriptional state; therefore, association of PRC1 components with enzymes involved in the removal of pre-established chromatin marks would be not surprising (Figure 3). In agreement with this, VAL1 interacts with the HISTONE DEACETYLASE 19 (HDA19). Mutation in HDA19 resulted in increased levels of gene activation marks, such as histone $\mathrm{H} 3$ acetylation, histone $\mathrm{H} 4$ acetylation, and $\mathrm{H} 3 \mathrm{~K} 4 \mathrm{me} 3$, but decreased levels of H3K27me3 marks at seed maturation genes after germination (Zhou et al., 2013), suggesting VAL as a link between histone deacetylation and PcG-mediated repression of seed maturation genes (Calonje, 2014). Interestingly HDA6 and HDA19 act redundantly in the repression of embryonic properties following germination in Arabidopsis (Tanaka et al., 2008). Furthermore, it has been recently shown that the trimethyl-H3K4 demethylase PKDM7B/JMJ14 in vivo interacts with the PRC1 components EMF1 and AtBMI1 and that this interaction is crucial for regulation of $F T$ (Wang et al., 2014),. In this work, the authors propose how the transcription factor FLC expressed in leaf veins regulates $F T$ expression ( $\mathrm{Gu}$ et al., 2013) by recruiting the vascular EMF1/JMJ14/AtBMI1 containing PRC1 to the $F T$ locus. Therefore, these data strongly support the association of this type of enzymes with PRC1. 


\section{Future directions}

Recent advances have improved our understanding on PRC1 function in plants; however, there is much that remains to be investigated. Unraveling the precise recruitment mechanism actually used by the cell, under which circumstances and in order to achieve which biological outcomes, remains one of the major challenges in the field. The identification of new PRC1 interacting factors and the characterization of plant PRC1 variants will allow us to go further in this direction. In addition, determining whether PRC1 plays roles beyond its classical repressive function might reveal new twists in PRC1-mediated gene regulation.

Another important question to be address concerns the functional role of the H2Aub mark and how it contributes to transcriptional repression. The distribution of H2Aub in the Arabidopsis genome it is not yet known, neither its role in gene repression. H2Aub in animals has been reported to interfere with the elongation of transcription by RNA Polymerase II (Zhou et al., 2008). Interestingly, a recent work indicates that blocking RNA Polymerase II transcription in mouse embryonic stem cells is sufficient to induce ectopic recruitment of $\mathrm{PRC} 2$ to $\mathrm{CpG}$ islands (Riising et al., 2014). An intriguing possibility would be that $\mathrm{H} 2 \mathrm{Aub}$ marks mediate the blocking of transcription, and that this induces PRC2 recruitment. A better understanding of the localization of H2Aub marks and the interplay of this mark with the transcriptional machinery will shed some light on the effect of this modification.

Finally, to understand the biological roles of PRC1, it will be important to determine whether the different $\mathrm{H} 2 \mathrm{~A}$ variants are susceptible to be monoubiquitinated and in that case, if the different PRC1 RING fingers exhibit target preferences.

\section{Acknowledgements}


This work is supported by Marie Curie CIG Grant ID 333748 and BIO2013-44078-P

Grant from the Spanish Ministry of Economy and Competitiveness (MINECO). We declare no conflict of interest.

\section{References}

Aichinger, E., Villar, C.B.R., Farrona, S., Reyes, J.C., Hennig, L. and Köhler, C. (2009) CHD3 proteins and polycomb group proteins antagonistically determine cell identity in Arabidopsis. PLoS Genet., 5, e1000605.

Aubert, D., Chen, L., Moon, Y.H., Martin, D., Castle, L.A., Yang, C.H. and Sung, Z.R. (2001) EMF1, a novel protein involved in the control of shoot architecture and flowering in Arabidopsis. Plant Cell, 13, 1865-1875.

Beh, L.Y., Colwell, L.J. and Francis, N.J. (2012) A core subunit of Polycomb repressive complex 1 is broadly conserved in function but not primary sequence. Proc. Natl. Acad. Sci. U. S. A., 109, E1063-1071.

Bentley, M.L., Corn, J.E., Dong, K.C., Phung, Q., Cheung, T.K. and Cochran, A.G. (2011) Recognition of UbcH5c and the nucleosome by the Bmi1/Ring1b ubiquitin ligase complex. EMBO J., 30, 3285-3297.

Bezsonova, I., Walker, J.R., Bacik, J.P., Duan, S., Dhe-Paganon, S. and Arrowsmith, C.H. (2009) Ring1B contains a ubiquitin-like docking module for interaction with Cbx proteins. Biochemistry (Mosc.), 48, 10542-10548.

Blackledge, N.P., Farcas, A.M., Kondo, T., et al. (2014) Variant PRC1 complexdependent $\mathrm{H} 2 \mathrm{~A}$ ubiquitylation drives $\mathrm{PRC} 2$ recruitment and polycomb domain formation. Cell, 157, 1445-1459. 
Bratzel, F., López-Torrejón, G., Koch, M., Pozo, J.C. Del and Calonje, M. (2010) Keeping cell identity in Arabidopsis requires PRC1 RING-finger homologs that catalyze H2A monoubiquitination. Curr. Biol. CB, 20, 1853-1859.

Bratzel, F., Yang, C., Angelova, A., López-Torrejón, G., Koch, M., Pozo, J.C. del and Calonje, M. (2012) Regulation of the new Arabidopsis imprinted gene AtBMI1C requires the interplay of different epigenetic mechanisms. Mol. Plant, 5, 260-269.

Buchwald, G., Stoop, P. van der, Weichenrieder, O., Perrakis, A., Lohuizen, M. van and Sixma, T.K. (2006) Structure and E3-ligase activity of the Ring-Ring complex of polycomb proteins Bmi1 and Ring1b. EMBO J., 25, 2465-2474.

Calonje, M. (2014) PRC1 marks the difference in plant PcG repression. Mol. Plant, 7, $459-471$.

Calonje, M., Sanchez, R., Chen, L. and Sung, Z.R. (2008) EMBRYONIC FLOWER1 participates in polycomb group-mediated AG gene silencing in Arabidopsis. Plant Cell, 20, 277-291.

Cao, R., Wang, L., Wang, H., Xia, L., Erdjument-Bromage, H., Tempst, P., Jones, R.S. and Zhang, Y. (2002) Role of histone H3 lysine 27 methylation in Polycomb-group silencing. Science, 298, 1039-1043.

Chanvivattana, Y., Bishopp, A., Schubert, D., Stock, C., Moon, Y.-H., Sung, Z.R. and Goodrich, J. (2004) Interaction of Polycomb-group proteins controlling flowering in Arabidopsis. Dev. Camb. Engl., 131, 5263-5276. 
Chen, D., Molitor, A., Liu, C. and Shen, W.-H. (2010) The Arabidopsis PRC1-like ring-finger proteins are necessary for repression of embryonic traits during vegetative growth. Cell Res., 20, 1332-1344.

Chen, L., Cheng, J.C., Castle, L. and Sung, Z.R. (1997) EMF genes regulate Arabidopsis inflorescence development. Plant Cell, 9, 2011-2024.

Comet, I. and Helin, K. (2014) Revolution in the Polycomb hierarchy. Nat. Struct. Mol. Biol., 21, 573-575.

Cooper, S., Dienstbier, M., Hassan, R., et al. (2014) Targeting polycomb to pericentric heterochromatin in embryonic stem cells reveals a role for H2AK119u1 in PRC2 recruitment. Cell Rep., 7, 1456-1470.

Czermin, B., Melfi, R., McCabe, D., Seitz, V., Imhof, A. and Pirrotta, V. (2002) Drosophila enhancer of Zeste/ESC complexes have a histone H3 methyltransferase activity that marks chromosomal Polycomb sites. Cell, 111, 185-196.

Derkacheva, M., Steinbach, Y., Wildhaber, T., Mozgová, I., Mahrez, W., Nanni, P., Bischof, S., Gruissem, W. and Hennig, L. (2013) Arabidopsis MSI1 connects LHP1 to PRC2 complexes. EMBO J., 32, 2073-2085.

Exner, V., Aichinger, E., Shu, H., Wildhaber, T., Alfarano, P., Caflisch, A., Gruissem, W., Köhler, C. and Hennig, L. (2009) The chromodomain of LIKE HETEROCHROMATIN PROTEIN 1 is essential for H3K27me3 binding and function during Arabidopsis development. PloS One, 4, e5335. 
Farcas, A.M., Blackledge, N.P., Sudbery, I., et al. (2012) KDM2B links the Polycomb Repressive Complex 1 (PRC1) to recognition of $\mathrm{CpG}$ islands. eLife, 1, e00205.

Francis, N.J., Kingston, R.E. and Woodcock, C.L. (2004) Chromatin compaction by a polycomb group protein complex. Science, 306, 1574-1577.

Francis, N.J., Saurin, A.J., Shao, Z. and Kingston, R.E. (2001) Reconstitution of a functional core polycomb repressive complex. Mol. Cell, 8, 545-556.

Gao, Z., Zhang, J., Bonasio, R., Strino, F., Sawai, A., Parisi, F., Kluger, Y. and Reinberg, D. (2012) PCGF homologs, CBX proteins, and RYBP define functionally distinct PRC1 family complexes. Mol. Cell, 45, 344-356.

Gaudin, V., Libault, M., Pouteau, S., Juul, T., Zhao, G., Lefebvre, D. and Grandjean, O. (2001) Mutations in LIKE HETEROCHROMATIN PROTEIN 1 affect flowering time and plant architecture in Arabidopsis. Dev. Camb. Engl., 128, 4847-4858.

Gil, J. and O'Loghlen, A. (2014) PRC1 complex diversity: where is it taking us? Trends Cell Biol., 24, 632-641.

Goodrich, J., Puangsomlee, P., Martin, M., Long, D., Meyerowitz, E.M. and Coupland, G. (1997) A Polycomb-group gene regulates homeotic gene expression in Arabidopsis. Nature, 386, 44-51.

Grau, D.J., Chapman, B.A., Garlick, J.D., Borowsky, M., Francis, N.J. and Kingston, R.E. (2011) Compaction of chromatin by diverse Polycomb group proteins requires localized regions of high charge. Genes Dev., 25, 2210-2221. 
Guerriero, G., Martin, N., Golovko, A., Sundström, J.F., Rask, L. and Ezcurra, I. (2009) The RY/Sph element mediates transcriptional repression of maturation genes from late maturation to early seedling growth. New Phytol., 184, 552-565.

Gutiérrez, L., Oktaba, K., Scheuermann, J.C., Gambetta, M.C., Ly-Hartig, N. and Müller, J. (2012) The role of the histone H2A ubiquitinase Sce in Polycomb repression. Dev. Camb. Engl., 139, 117-127.

Gu, X., Le, C., Wang, Y., Li, Z., Jiang, D., Wang, Y. and He, Y. (2013) Arabidopsis FLC clade members form flowering-repressor complexes coordinating responses to endogenous and environmental cues. Nat. Commun., 4, 1947.

He, J., Shen, L., Wan, M., Taranova, O., Wu, H. and Zhang, Y. (2013) Kdm2b maintains murine embryonic stem cell status by recruiting PRC1 complex to CpG islands of developmental genes. Nat. Cell Biol., 15, 373-384.

Hennig, L. and Derkacheva, M. (2009) Diversity of Polycomb group complexes in plants: same rules, different players? Trends Genet. TIG, 25, 414-423.

Hoppmann, V., Thorstensen, T., Kristiansen, P.E., Veiseth, S.V., Rahman, M.A., Finne, K., Aalen, R.B. and Aasland, R. (2011) The CW domain, a new histone recognition module in chromatin proteins. EMBO J., 30, 1939-1952.

Kalb, R., Latwiel, S., Baymaz, H.I., Jansen, P.W.T.C., Müller, C.W., Vermeulen, M. and Müller, J. (2014) Histone H2A monoubiquitination promotes histone H3 methylation in Polycomb repression. Nat. Struct. Mol. Biol., 21, 569-571. 
Kim, S.Y., Lee, J., Eshed-Williams, L., Zilberman, D. and Sung, Z.R. (2012) EMF1 and PRC2 cooperate to repress key regulators of Arabidopsis development. PLoS Genet., 8, e1002512.

Kim, S.Y., Zhu, T. and Sung, Z.R. (2010) Epigenetic regulation of gene programs by EMF1 and EMF2 in Arabidopsis. Plant Physiol., 152, 516-528.

Klymenko, T., Papp, B., Fischle, W., Köcher, T., Schelder, M., Fritsch, C., Wild, B., Wilm, M. and Müller, J. (2006) A Polycomb group protein complex with sequence-specific DNA-binding and selective methyl-lysine-binding activities. Genes Dev., 20, 1110-1122.

Köhler, C., Hennig, L., Bouveret, R., Gheyselinck, J., Grossniklaus, U. and Gruissem, W. (2003) Arabidopsis MSI1 is a component of the MEA/FIE Polycomb group complex and required for seed development. EMBO J., 22, $4804-4814$.

Köhler, C., Hennig, L., Spillane, C., Pien, S., Gruissem, W. and Grossniklaus, U. (2003) The Polycomb-group protein MEDEA regulates seed development by controlling expression of the MADS-box gene PHERES1. Genes Dev., 17, $1540-1553$.

Kondo, T., Isono, K., Kondo, K., Endo, T.A., Itohara, S., Vidal, M. and Koseki, H. (2014) Polycomb potentiates meis2 activation in midbrain by mediating interaction of the promoter with a tissue-specific enhancer. Dev. Cell, 28, 94101.

Kotake, T., Takada, S., Nakahigashi, K., Ohto, M. and Goto, K. (2003) Arabidopsis TERMINAL FLOWER 2 gene encodes a heterochromatin protein 1 homolog 
and represses both FLOWERING LOCUS $\mathrm{T}$ to regulate flowering time and several floral homeotic genes. Plant Cell Physiol., 44, 555-564.

Lagarou, A., Mohd-Sarip, A., Moshkin, Y.M., Chalkley, G.E., Bezstarosti, K., Demmers, J.A.A. and Verrijzer, C.P. (2008) dKDM2 couples histone H2A ubiquitylation to histone H3 demethylation during Polycomb group silencing. Genes Dev., 22, 2799-2810.

Lee, W.Y., Lee, D., Chung, W.-I. and Kwon, C.S. (2009) Arabidopsis ING and Alfin1-like protein families localize to the nucleus and bind to $\mathrm{H} 3 \mathrm{~K} 4 \mathrm{me} 3 / 2$ via plant homeodomain fingers. Plant J. Cell Mol. Biol., 58, 511-524.

Levine, S.S., King, I.F.G. and Kingston, R.E. (2004) Division of labor in polycomb group repression. Trends Biochem. Sci., 29, 478-485.

Levine, S.S., Weiss, A., Erdjument-Bromage, H., Shao, Z., Tempst, P. and Kingston, R.E. (2002) The core of the polycomb repressive complex is compositionally and functionally conserved in flies and humans. Mol. Cell. Biol., 22, 6070-6078.

Libault, M., Tessadori, F., Germann, S., Snijder, B., Fransz, P. and Gaudin, V. (2005) The Arabidopsis LHP1 protein is a component of euchromatin. Planta, 222, 910-925.

Li, W., Wang, Z., Li, J., Yang, H., Cui, S., Wang, X. and Ma, L. (2011) Overexpression of AtBMI1C, a polycomb group protein gene, accelerates flowering in Arabidopsis. PloS One, 6, e21364. 
Li, Z., Cao, R., Wang, M., Myers, M.P., Zhang, Y. and Xu, R.-M. (2006) Structure of a Bmi-1-Ring1B polycomb group ubiquitin ligase complex. J. Biol. Chem., 281, 20643-20649.

Lopez-Vernaza, M., Yang, S., Müller, R., Thorpe, F., Leau, E. de and Goodrich, J. (2012) Antagonistic roles of SEPALLATA3, FT and FLC genes as targets of the polycomb group gene CURLY LEAF. PloS One, 7, e30715.

Lo, S.M. and Francis, N.J. (2010) Inhibition of chromatin remodeling by polycomb group protein posterior sex combs is mechanistically distinct from nucleosome binding. Biochemistry (Mosc.), 49, 9438-9448.

March-Díaz, R. and Reyes, J.C. (2009) The beauty of being a variant: H2A.Z and the SWR1 complex in plants. Mol. Plant, 2, 565-577.

Margueron, R. and Reinberg, D. (2011) The Polycomb complex PRC2 and its mark in life. Nature, 469, 343-349.

McGinty, R.K., Henrici, R.C. and Tan, S. (2014) Crystal structure of the PRC1 ubiquitylation module bound to the nucleosome. Nature, 514, 591-596.

Molitor, A.M., Bu, Z., Yu, Y. and Shen, W.-H. (2014) Arabidopsis AL PHD-PRC1 complexes promote seed germination through H3K4me3-to-H3K27me3 chromatin state switch in repression of seed developmental genes. PLoS Genet., 10, e1004091.

Moon, Y.-H., Chen, L., Pan, R.L., Chang, H.-S., Zhu, T., Maffeo, D.M. and Sung, Z.R. (2003) EMF genes maintain vegetative development by repressing the flower program in Arabidopsis. Plant Cell, 15, 681-693. 
Morey, L., Pascual, G., Cozzuto, L., Roma, G., Wutz, A., Benitah, S.A. and Croce, L. Di (2012) Nonoverlapping functions of the Polycomb group Cbx family of proteins in embryonic stem cells. Cell Stem Cell, 10, 47-62.

Müller, J., Hart, C.M., Francis, N.J., et al. (2002) Histone methyltransferase activity of a Drosophila Polycomb group repressor complex. Cell, 111, 197-208.

Müller, J. and Müller, C.W. (2014) Structural biology: Enzyme-chromatin complex visualized. Nature, 514, 572-573.

Nakahigashi, K., Jasencakova, Z., Schubert, I. and Goto, K. (2005) The Arabidopsis heterochromatin protein1 homolog (TERMINAL FLOWER2) silences genes within the euchromatic region but not genes positioned in heterochromatin. Plant Cell Physiol., 46, 1747-1756.

Napoles, M. de, Mermoud, J.E., Wakao, R., et al. (2004) Polycomb group proteins Ring1A/B link ubiquitylation of histone $\mathrm{H} 2 \mathrm{~A}$ to heritable gene silencing and $\mathrm{X}$ inactivation. Dev. Cell, 7, 663-676.

Park, H.-Y., Lee, S.-Y., Seok, H.-Y., Kim, S.-H., Sung, Z.R. and Moon, Y.-H. (2011) EMF1 interacts with EIP1, EIP6 or EIP9 involved in the regulation of flowering time in Arabidopsis. Plant Cell Physiol., 52, 1376-1388.

Peterson, A.J., Mallin, D.R., Francis, N.J., Ketel, C.S., Stamm, J., Voeller, R.K., Kingston, R.E. and Simon, J.A. (2004) Requirement for sex comb on midleg protein interactions in Drosophila polycomb group repression. Genetics, 167, 1225-1239. 
Riising, E.M., Comet, I., Leblanc, B., Wu, X., Johansen, J.V. and Helin, K. (2014) Gene silencing triggers polycomb repressive complex 2 recruitment to $\mathrm{CpG}$ islands genome wide. Mol. Cell, 55, 347-360.

Sanchez-Pulido, L., Devos, D., Sung, Z.R. and Calonje, M. (2008) RAWUL: a new ubiquitin-like domain in PRC1 ring finger proteins that unveils putative plant and worm PRC1 orthologs. BMC Genomics, 9, 308.

Sanchez, R. and Zhou, M.-M. (2011) The PHD finger: a versatile epigenome reader. Trends Biochem. Sci., 36, 364-372.

Saurin, A.J., Shao, Z., Erdjument-Bromage, H., Tempst, P. and Kingston, R.E. (2001) A Drosophila Polycomb group complex includes Zeste and dTAFII proteins. Nature, 412, 655-660.

Schoorlemmer, J., Marcos-Gutiérrez, C., Were, F., Martínez, R., García, E., Satijn, D.P., Otte, A.P. and Vidal, M. (1997) Ring1A is a transcriptional repressor that interacts with the Polycomb-M33 protein and is expressed at rhombomere boundaries in the mouse hindbrain. EMBO J., 16, 5930-5942.

Schubert, D., Primavesi, L., Bishopp, A., Roberts, G., Doonan, J., Jenuwein, T. and Goodrich, J. (2006) Silencing by plant Polycomb-group genes requires dispersed trimethylation of histone $\mathrm{H} 3$ at lysine 27. EMBO J., 25, 4638-4649.

Schuettengruber, B. and Cavalli, G. (2009) Recruitment of polycomb group complexes and their role in the dynamic regulation of cell fate choice. Dev. Camb. Engl., 136, 3531-3542. 
Schwartz, Y.B. and Pirrotta, V. (2013) A new world of Polycombs: unexpected partnerships and emerging functions. Nat. Rev. Genet., 14, 853-864.

Sevilla, A. and Binda, O. (2014) Post-translational modifications of the histone variant H2AZ. Stem Cell Res., 12, 289-295.

Shao, Z., Raible, F., Mollaaghababa, R., Guyon, J.R., Wu, C.T., Bender, W. and Kingston, R.E. (1999) Stabilization of chromatin structure by PRC1, a Polycomb complex. Cell, 98, 37-46.

Shen, L., Thong, Z., Gong, X., Shen, Q., Gan, Y. and Yu, H. (2014) The putative PRC1 RING-finger protein AtRING1A regulates flowering through repressing MADS AFFECTING FLOWERING genes in Arabidopsis. Dev. Camb. Engl., 141, 1303-1312.

Simon, J.A. and Kingston, R.E. (2009) Mechanisms of polycomb gene silencing: knowns and unknowns. Nat. Rev. Mol. Cell Biol., 10, 697-708.

Sung, Z.R., Belachew, A., Shunong, B. and Bertrand-Garcia, R. (1992) EMF, an Arabidopsis Gene Required for Vegetative Shoot Development. Science, 258, $1645-1647$.

Sung, Z.R., Chen, L., Moon, Y.-H. and Lertpiriyapong, K. (2003) Mechanisms of floral repression in Arabidopsis. Curr. Opin. Plant Biol., 6, 29-35.

Suzuki, M. and McCarty, D.R. (2008) Functional symmetry of the B3 network controlling seed development. Curr. Opin. Plant Biol., 11, 548-553.

Suzuki, M., Wang, H.H.-Y. and McCarty, D.R. (2007) Repression of the LEAFY COTYLEDON 1/B3 regulatory network in plant embryo development by 
VP1/ABSCISIC ACID INSENSITIVE 3-LIKE B3 genes. Plant Physiol., 143, 902-911.

Tanaka, M., Kikuchi, A. and Kamada, H. (2008) The Arabidopsis histone deacetylases HDA6 and HDA19 contribute to the repression of embryonic properties after germination. Plant Physiol., 146, 149-161.

Tavares, L., Dimitrova, E., Oxley, D., et al. (2012) RYBP-PRC1 complexes mediate $\mathrm{H} 2 \mathrm{~A}$ ubiquitylation at polycomb target sites independently of PRC2 and H3K27me3. Cell, 148, 664-678.

Tonkin, E., Hagan, D.-M., Li, W. and Strachan, T. (2002) Identification and characterisation of novel mammalian homologues of Drosophila polyhomeoticpermits new insights into relationships between members of the polyhomeotic family. Hum. Genet., 111, 435-442.

Torok, M. and Etkin, L.D. (2001) Two B or not two B? Overview of the rapidly expanding B-box family of proteins. Differ. Res. Biol. Divers., 67, 63-71.

Turck, F., Fornara, F. and Coupland, G. (2008) Regulation and identity of florigen: FLOWERING LOCUS T moves center stage. Annu. Rev. Plant Biol., 59, 573594.

Turck, F., Roudier, F., Farrona, S., et al. (2007) Arabidopsis TFL2/LHP1 specifically associates with genes marked by trimethylation of histone H3 lysine 27. PLoS Genet., 3, e86. 
Wang, H., Wang, L., Erdjument-Bromage, H., Vidal, M., Tempst, P., Jones, R.S. and Zhang, Y. (2004) Role of histone H2A ubiquitination in Polycomb silencing. Nature, 431, 873-878.

Wang, Y., Gu, X., Yuan, W., Schmitz, R.J. and He, Y. (2014) Photoperiodic control of the floral transition through a distinct polycomb repressive complex. Dev. Cell, 28, 727-736.

Wu, X., Johansen, J.V. and Helin, K. (2013) Fbx110/Kdm2b recruits polycomb repressive complex 1 to $\mathrm{CpG}$ islands and regulates $\mathrm{H} 2 \mathrm{~A}$ ubiquitylation. Mol. Cell, 49, 1134-1146.

Xu, L. and Shen, W.-H. (2008) Polycomb silencing of KNOX genes confines shoot stem cell niches in Arabidopsis. Curr. Biol. CB, 18, 1966-1971.

Yang, C., Bratzel, F., Hohmann, N., Koch, M., Turck, F. and Calonje, M. (2013) VAL- and AtBMI1-mediated H2Aub initiate the switch from embryonic to postgerminative growth in Arabidopsis. Curr. Biol. CB, 23, 1324-1329.

Yang, C.H., Chen, L.J. and Sung, Z.R. (1995) Genetic regulation of shoot development in Arabidopsis: role of the EMF genes. Dev. Biol., 169, 421-435.

Zhang, K., Sridhar, V.V., Zhu, J., Kapoor, A. and Zhu, J.-K. (2007) Distinctive core histone post-translational modification patterns in Arabidopsis thaliana. PloS One, 2, e1210.

Zhang, X., Germann, S., Blus, B.J., Khorasanizadeh, S., Gaudin, V. and Jacobsen, S.E. (2007) The Arabidopsis LHP1 protein colocalizes with histone H3 Lys27 trimethylation. Nat. Struct. Mol. Biol., 14, 869-871. 
Zhou, W., Zhu, P., Wang, J., Pascual, G., Ohgi, K.A., Lozach, J., Glass, C.K. and Rosenfeld, M.G. (2008) Histone H2A monoubiquitination represses transcription by inhibiting RNA polymerase II transcriptional elongation. Mol. Cell, 29, 69-80.

Zhou, Y., Tan, B., Luo, M., et al. (2013) HISTONE DEACETYLASE19 interacts with HSL1 and participates in the repression of seed maturation genes in Arabidopsis seedlings. Plant Cell, 25, 134-148.

\section{Figure legends}

Figure 1. Schematic representation of the domain architecture of the PRC1 components in Drosophila, Arabidopsis and vertebrates. The characteristic domains of the different proteins are indicated. Ring: RING (Really Interesting New Gene) finger domain; RAWUL: Ring-finger and WD40 associated ubiquitin-like domain; ZF_FSC: MYM-type Zinc finger with FCS sequence motif; SAM: sterile $\alpha$-motif; CD: Chromodomain; PC: Pc-box; CDS: Chromo Shadow domain.

Figure 2. New model for recruitment of PcG complexes. PRC1 is targeted to genes through recruiting factors (Recruiters). The interaction of PRC1 with different Recruiters determines the type of repression: (1) H2A-dependent repression in which PRC1 mediates H2Aub to block transcription; or (2) H2A-independent repression in which PRC1 mediates chromatin compaction. In any case, PRC1 recruits PRC2 through a yet unknown mechanism to incorporate $\mathrm{H} 3 \mathrm{~K} 27 \mathrm{me} 3$ marks that maintains a stable repression. Yellow circles indicate H2Aub marks; Red triangles indicate H3K27me3 marks. 
Figure 3. PRC1 associated proteins in Arabidopsis. Three different types of factors have been shown to interact with Arabidopsis PRC1 core components until date. DNA binding factors, histone modification interacting factors and factors that remove previously established chromatin marks. Some of these properties can be present in the same protein, as is the case of the VAL factors. The domains involved in the different activities are indicated in parenthesis. 


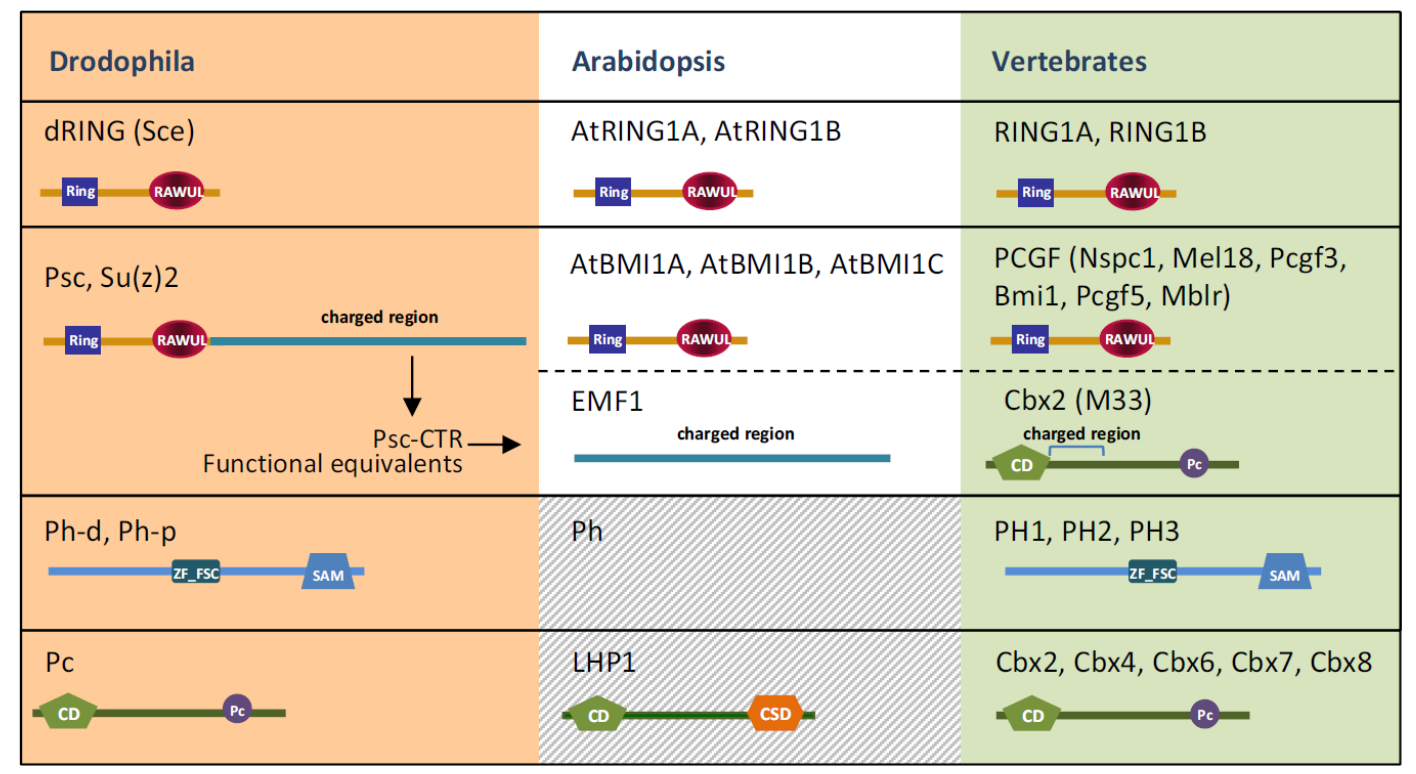

Figure 1. Schematic representation of the domain architecture of the PRC1 components in Drosophila, Arabidopsis and vertebrates. The characteristic domains of the different proteins are indicated. Ring: RING (Really Interesting New Gene) finger domain; RAWUL: Ring-finger and WD40 associated ubiquitin-like domain; ZF_FSC: MYM-type Zinc finger with FCS sequence motif; SAM: sterile a-motif; CD: Chromodomain; PC: Pc-box; CDS: Chromo Shadow domain. 


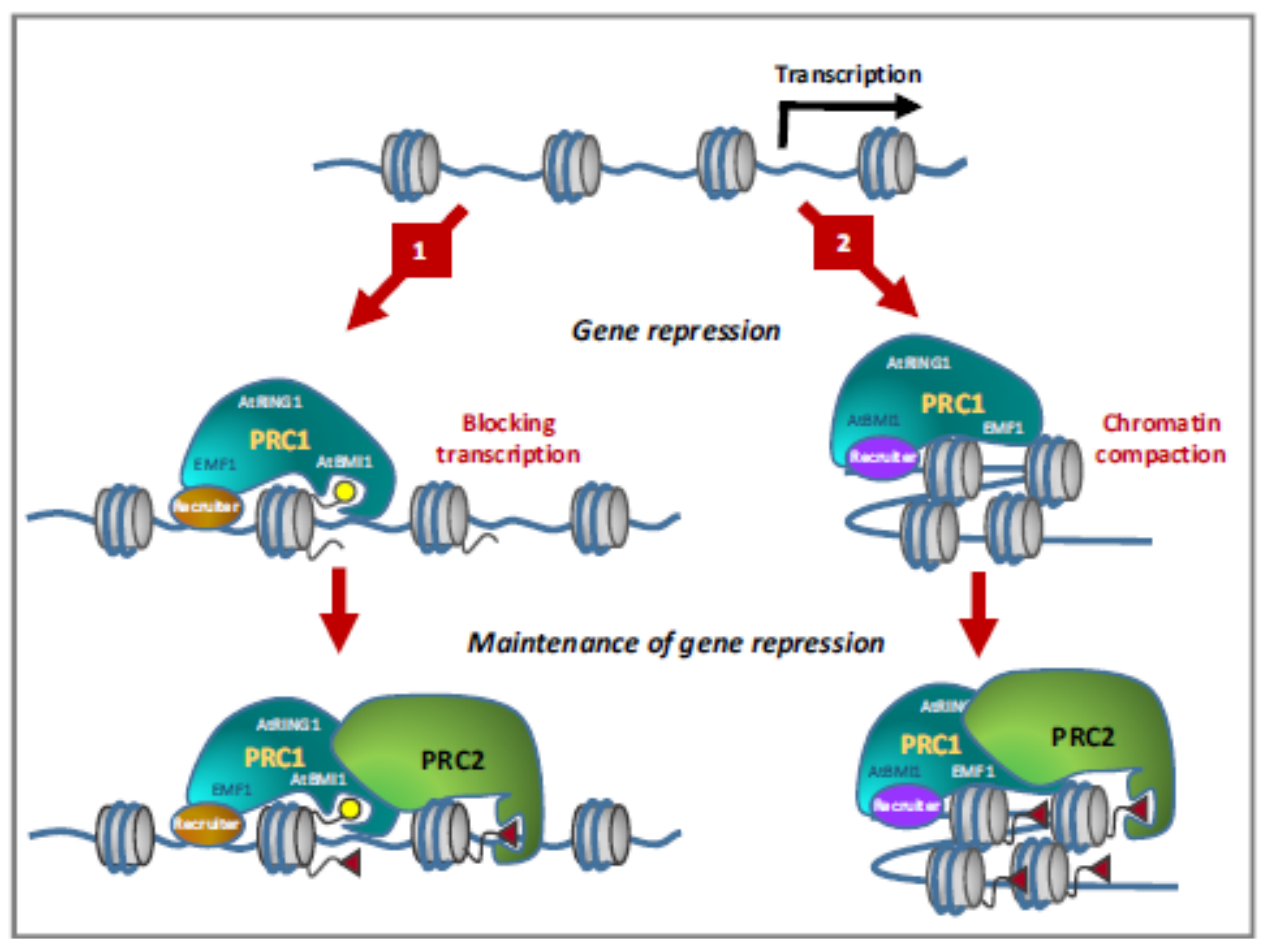

Figure 2. New model for recruitment of PcG complexes. PRC1 is targeted to genes through recruiting factors (Recruiters). The interaction of PRC1 with different Recruiters determines the type of repression: (1) H2A-dependent repression in which PRC1 mediates $\mathrm{H} 2 \mathrm{Aub}$ to block transcription; or (2) H2A-independent repression in which PRC1 mediates chromatin compaction. In any case, PRC1 recruits PRC2 through a yet unknown mechanism to incorporate $\mathrm{H} 3 \mathrm{~K} 27 \mathrm{me} 3$ marks that maintains a stable repression. Yellow circles indicate H2Aub marks; Red triangles indicate $\mathrm{H} 3 \mathrm{~K} 27$ me 3 marks. 


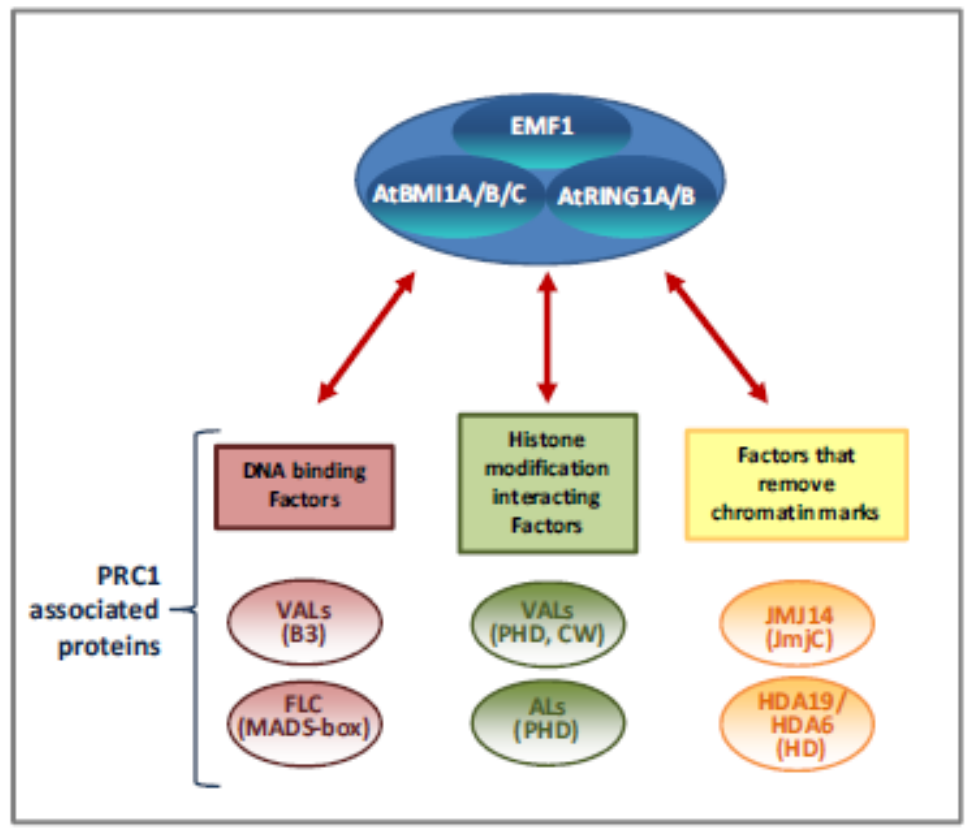

Figure 3. PRC1 associated proteins in Arabidopsis. Three different types of factors have been shown to interact with Arabidopsis PRC1 core components until date. DNA binding factors, histone modification interacting factors and factors that remove previously established chromatin marks. Some of these properties can be present in the same protein, as is the case of the VAL factors. The domains involved in the different activities are indicated in parenthesis. 